ARTICLE

DOI: $10.1038 / s 41467-018-04630-w$

\title{
Near infrared light induced plasmonic hot hole transfer at a nano-heterointerface
}

\author{
Zichao Lian (1) 1, Masanori Sakamoto², Hironori Matsunaga ${ }^{3}$, Junie Jhon M. Vequizo ${ }^{3}$, Akira Yamakata (i) $^{3}$, \\ Mitsutaka Haruta (iD ${ }^{2}$, Hiroki Kurata ${ }^{2}$, Wataru Ota ${ }^{4}$, Tohru Sato ${ }^{4,5,6}$ \& Toshiharu Teranishi ${ }^{2}$
}

Localized surface plasmon resonance (LSPR)-induced hot-carrier transfer is a key mechanism for achieving artificial photosynthesis using the whole solar spectrum, even including the infrared (IR) region. In contrast to the explosive development of photocatalysts based on the plasmon-induced hot electron transfer, the hole transfer system is still quite immature regardless of its importance, because the mechanism of plasmon-induced hole transfer has remained unclear. Herein, we elucidate LSPR-induced hot hole transfer in CdS/CuS heterostructured nanocrystals (HNCs) using time-resolved IR (TR-IR) spectroscopy. TR-IR spectroscopy enables the direct observation of carrier in a LSPR-excited CdS/CuS HNC. The spectroscopic results provide insight into the novel hole transfer mechanism, named plasmon-induced transit carrier transfer (PITCT), with high quantum yields (19\%) and longlived charge separations $(9.2 \mu \mathrm{s})$. As an ultrafast charge recombination is a major drawback of all plasmonic energy conversion systems, we anticipate that PITCT will break the limit of conventional plasmon-induced energy conversion.

\footnotetext{
${ }^{1}$ Department of Chemistry, Graduate School of Science, Kyoto University, Gokasho, Uji, Kyoto 611-0011, Japan. ${ }^{2}$ Institute for Chemical Research, Kyoto University, Gokasho, Uji, Kyoto 611-0011, Japan. ${ }^{3}$ Graduate School of Engineering, Toyota Technological Institute, 2-12-1 Hisakata, Tempaku, Nagoya 4688511, Japan. ${ }^{4}$ Department of Molecular Engineering, Graduate School of Engineering, Kyoto University, Nishikyo-ku, Kyoto 615-8510, Japan. ${ }^{5}$ Fukui Institute for Fundamental Chemistry, Kyoto University, Takano-Nishihiraki-cho 34-4, Sakyo-ku, Kyoto 606-8103, Japan. ${ }^{6}$ Unit of Elements Strategy Initiative for Catalysts and Batteries, Kyoto University, Nishikyo-ku, Kyoto 615-8510, Japan. Correspondence and requests for materials should be addressed to M.S. (email: sakamoto@scl.kyoto-u.ac.jp) or to T.T. (email: teranisi@scl.kyoto-u.ac.jp)
} 
ocalized surface plasmon resonance (LSPR)-induced photoenergy conversion is among the great challenges causing a paradigm shift in both scientific fields and industry regarding solar-energy utilization ${ }^{1-5}$. The LSPR band can be tuned over a wide spectral range by changing the carrier density, morphology, and other material properties, enabling solar energy utilization from ultraviolet (UV) to infrared (IR) regions. Although plasmonic materials have superior light-harvesting abilities, the low conversion efficiency caused by ultrafast relaxation of the hot carrier and charge recombination is a major drawback. Hot carrier transfer competes with ultrafast relaxation via carrier scattering with timescales of just hundreds of femtoseconds (fs) ${ }^{6,7}$, and recombination after the charge separation completes in picoseconds (ps) region. Therefore, it is difficult to achieve sufficient extraction of hot carriers for practical applications from the kinetic perspective ${ }^{8,9}$. Furthermore, the unclear behavior of hot holes, which has remained an obscure subject regardless of the unmistakable importance, becomes an obstacle for the comprehensive understanding of LSPR-induced carrier transfer.

Recently, a novel series of compound semiconductors, copper chalcogenide nanocrystals, which show excellent tunable holebased LSPR absorption in the near-IR (NIR) region, have attracted much attention as candidates for IR-responsive photocatalysts $^{10,11}$. For heterostructured nanocrystals (HNCs) composed of plasmonic copper sulfide (CuS) phase and another metal or semiconductor phase (for example, acceptor phase), hot hole transfer has been proposed as a possible mechanism for providing IR-induced catalytic activity. However, the mechanism has yet to be determined. Elucidation of this mechanism would clarify the role of hot holes contributing the photocatalytic activity $^{12}$.

Herein, we elucidate the LSPR-induced behavior of hot holes in $\mathrm{CuS}$ NCs and CdS/CuS HNCs using time-resolved infrared (TRIR) spectroscopy. TR-IR spectroscopy enables us the direct observation of carrier in a photo-excited nanocrystal. The CdS/ $\mathrm{CuS} \mathrm{HNCs}$ are one of the promising combinations for the spectroscopic tracing of the LSPR-induced hole transfer from the CuS phase to the CdS phase. We discover in the detailed investigation that a multi-step carrier transfer (plasmon-induced transit carrier transfer: PITCT) realized efficient hole transfer from the CuS phase to the CdS phase. Surprisingly, the PITCT of CdS/CuS HNCs achieves high quantum yields (19\%) and long-lived charge separations $(9.2 \mu \mathrm{s})$, which has not been observed in plasmoninduced carrier-injection systems. Because ultrafast charge recombination is a major drawback of all plasmonic energy conversion systems, the PITCT mechanism proposes here should change the conventional consensus regarding LSPR-induced energy conversion due to the overwhelming advantage of high hot carrier transfer efficiency caused by in situ trapping of hot carriers and long-lived charge separation.

\section{Results}

Characterization of materials. We synthesized CdS/CuS HNCs using plate-shaped CuS NCs as seeds (see details in the Supplementary Figs. 1-5 and Supplementary Note 1). Figure 1a shows transmission electron microscopy (TEM) images of monodisperse CuS NCs (size, $16.3 \pm 1.5 \mathrm{~nm}$; thickness, $5.7 \pm 1.1 \mathrm{~nm}$ ). When CdS phases were grown on the CuS NCs, multiple CdS satellites of $3.8 \pm 0.8 \mathrm{~nm}$ in size were deposited on the peripheral regions of the CuS NCs, as shown in Fig. 1b. The X-ray diffraction (XRD) patterns in Fig. If clearly show that the CdS/CuS HNCs were composed of hexagonal covellite $\mathrm{CuS}(c v$-CuS, Joint Committee on Power Diffraction Standards (JCPDS) no. 06-0464) and wurtzite CdS ( $w$-CdS, JCPDS no. 89-944) phases, with the $\mathrm{Cd} / \mathrm{Cu}$ molar ratio determined as $38: 62$ by X-ray fluorescence (XRF) spectroscopy.

The high-resolution TEM (HRTEM) image in Fig. 1c shows the high crystallinity of the CdS/CuS HNCs and the different lattice fringes corresponding to $w$-CdS $(10-10)$ and $c v$-CuS (10-10) lattices. The fast Fourier transform (FFT) of Fig. 1c shows splitting spots that correspond to the $\mathrm{CdS}$ and $\mathrm{CuS}$ phases (Fig. 1d) and are aligned toward the same radial direction from the central spot, indicating the epitaxial growth of CdS on the $\mathrm{CuS}$ phase. High-angle annular dark-field (HAADF) scanning TEM (STEM) (Fig. 1e) and STEM-energy dispersive X-ray spectrometry (EDS) mapping (Fig. 1g) also showed that the $\mathrm{CuS}$ NCs were surrounded by multiple CdS satellites.

To clarify the atomic arrangement at the interface between $w$ $\mathrm{CdS}$ and $c v$-CuS, HAADF-STEM measurements were carried out as shown in Fig. 1h, $\mathrm{i}^{13}$. In the HAADF-STEM image, since only $\mathrm{Cd}$ and $\mathrm{Cu}$ atomic columns in $w$-CdS and $c v$-CuS were clearly observed but $\mathrm{S}$ columns were not observed, it is impossible to distinguish the polarity. The CuS and CdS phases had orientation relationships of $w$-CdS [0001]//cv-CuS [0001] and $w$-CdS [11-20]//cv-CuS [11-20], which resulted in intimate contact between the two phases. It is considered that $c v$-CuS (0001) and (000-1) planes were terminated by $S$ anion from the view point of the atomic arrangement of $\mathrm{Cu}$ ion. And it seems that $\mathrm{S}$ anions are connected to $\mathrm{Cd}^{2+}$-terminated $w$-CdS (0001) planes with a lattice mismatch of $8.33 \%$ and $w$-CdS phases are epitaxially grown on the $c v$-CuS NCs to form CdS/CuS HNCs.

Optical properties of materials and TR-IR measurements. Next, we investigated LSPR-induced hole transfer from the CuS phase to the CdS phase. Figure 2a shows the extinction spectra of CuS NCs and CdS/CuS HNCs (see also Supplementary Fig. 1). The CuS NCs showed an LSPR peak at $1080 \mathrm{~nm}$, while the LSPR peak of the CdS/CuS HNCs was red-shifted to $1254 \mathrm{~nm}$. This redshift of the LSPR peak might be attributed to the change of size, shape, and different dielectric environment with the existence of CdS phases ${ }^{14}$. Band diagrams of the CuS NCs and CdS NCs (Fig. 2b, Supplementary Figs. 6 and 7 for estimations of the conduction band (CB) and valence band (VB) positions) showed that the VB edge of the CdS NCs was $0.73 \mathrm{eV}$ lower than the Fermi level of the CuS NCs $\left(E_{\mathrm{pF}}\right)$, and could be accessed by hot holes generated by NIR LSPR in the CuS phases.

We conducted TR-IR measurements of CuS NCs and CdS/CuS HNCs in the $\mu$ s region to investigate the LSPR-induced hole dynamics. Figure $2 \mathrm{c}$ and $\mathrm{d}$ show the TR-IR absorption spectra of the $\mathrm{CuS}$ NCs and $\mathrm{CdS} / \mathrm{CuS} \mathrm{HNCs}$ in the $\mu$ s region after excitation of hole-based LSPR in the CuS phases with a 1200-nm laser (1.03 $\mathrm{eV})$ to produce hot holes. The TR-IR spectra of the CuS NCs showed bleaching peaks at around $740 \mathrm{~nm}$. In contrast, the TR-IR spectra of the CdS/CuS HNCs showed bleaching peaks at around $840 \mathrm{~nm}$ and absorption in the visible region. These transient absorption (TA) signals completely recovered within $1 \mathrm{~ms}$ after excitation, indicating that the observed spectral changes reflected a reversible process. As the LSPR-excitation-induced sequential event (that is, hole dephasing, hole-hole scattering, hole-phonon coupling, and lattice heat dissipation) was completed in the ps-ns region $3,15,16$, the observed spectral changes cannot be explained by the conventional LSPR-induced decay process. Furthermore, the bleaching peak was significantly blue-shifted compared with the LSPR peak. Therefore, we considered that this TR-IR spectral change originated from the reduction in the number of holes in the intrinsic state by trapping.

As the LSPR of compound semiconductors, which have a smaller number of carriers than metals, are sensitive to changes in carrier density, the redistribution of holes by trapping would 

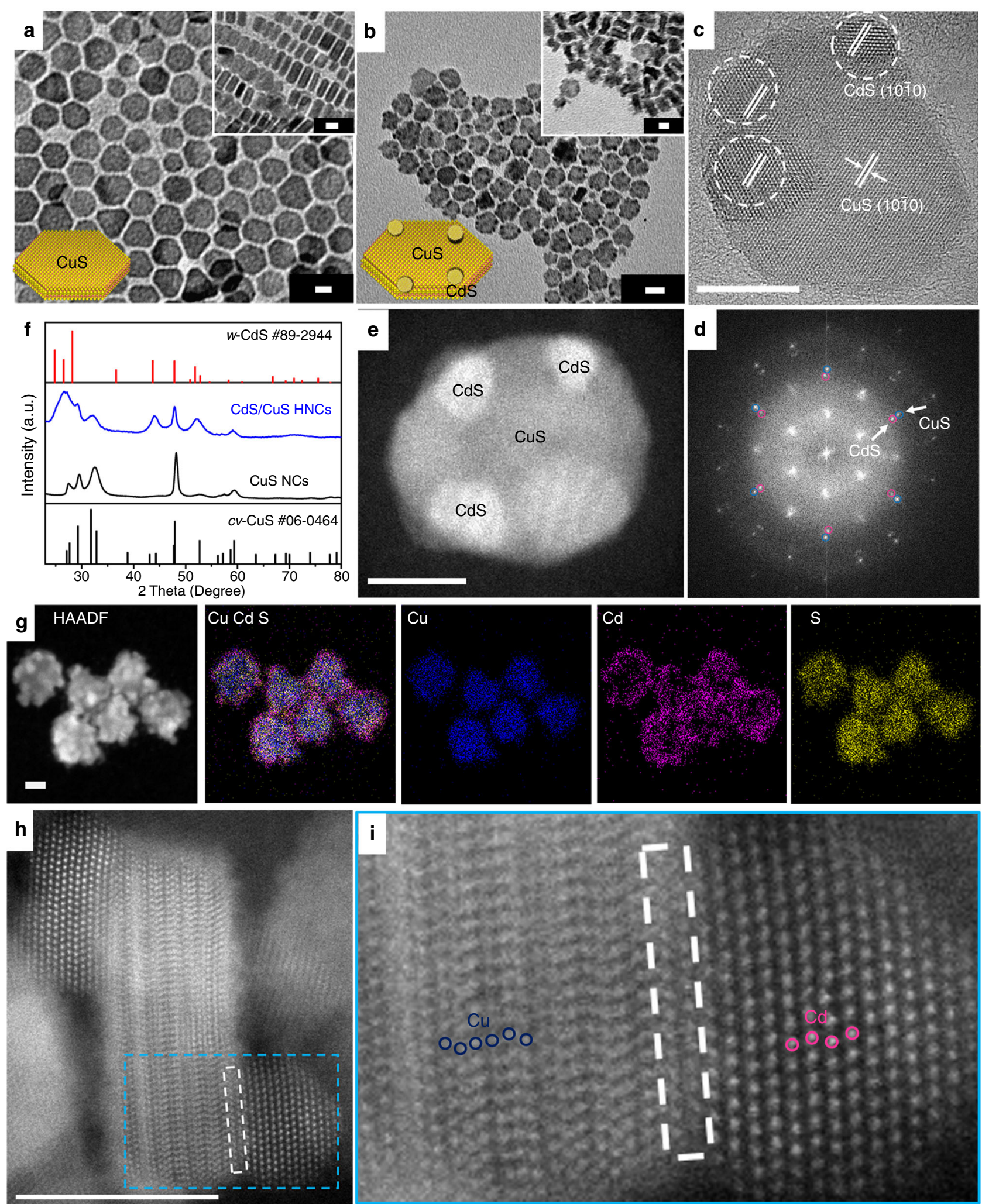

Fig. 1 Structural characterization of the nanocrystals. a, b Representative transmission electron microscopy (TEM) images of a plate-shaped CuS nanocrystals (NCs) (inset: stacked CuS NCs), b CdS/CuS herostructured NCs (HNCs) (inset: stacked CdS/CuS HNCs). c High-resolution TEM (HRTEM) image of CdS/CuS HNCs from the $c$ axis. d Fast Fourier transform (FFT) patterns of a single CdS/CuS HNC from c. e High-angle annular dark-field scanning TEM (HAADF-STEM) image of a single CdS/CuS HNC. $\mathbf{f}$ X-ray diffraction (XRD) patterns of CuS NCs and CdS/CuS HNCs. $\mathbf{g}$ HAADF-STEM energy dispersive X-ray spectrometry (EDS) elemental mapping images of CdS/CuS HNCs. h HAADF-STEM image of stacked CdS/CuS HNCs, where the interface is labeled with a dashed white rectangle and the electron beam incident direction is parallel to [11-20]. $\mathbf{i}$ Enlarged part of $\mathbf{h}$ with a dashed blue rectangle, showing $\mathrm{Cu}$ and $\mathrm{Cd}$ atomic arrangements. Scale bars: $10 \mathrm{~nm}$ 
a
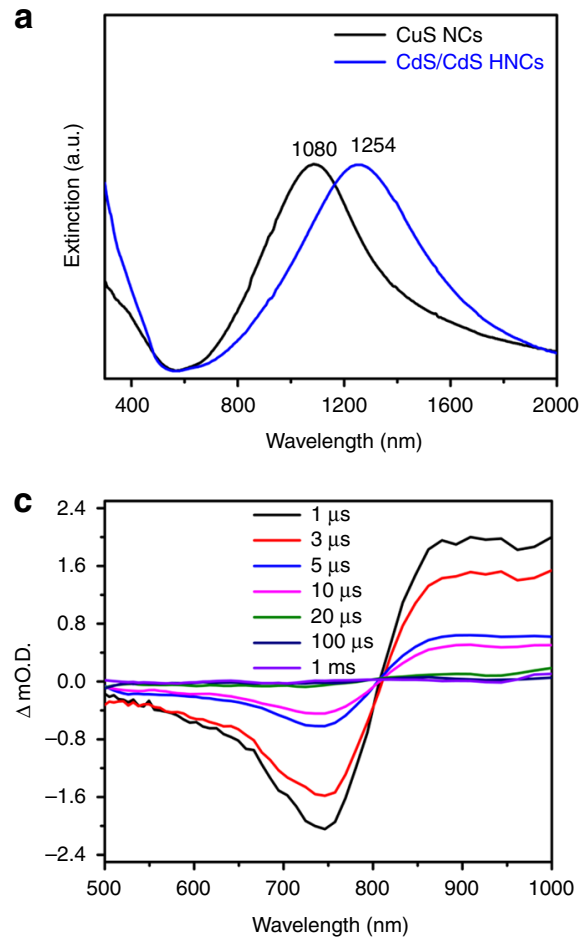

e

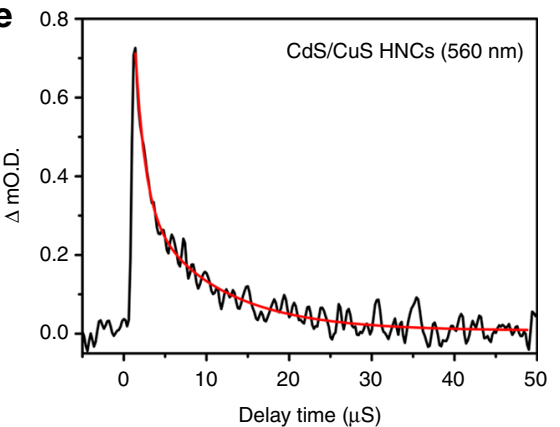

b

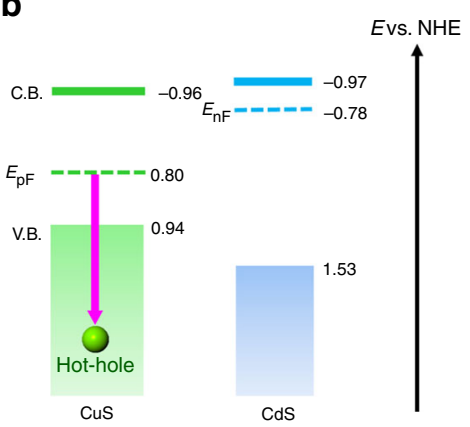

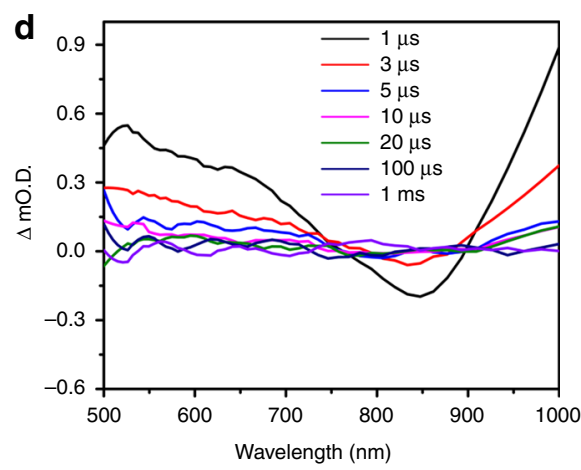

f

g
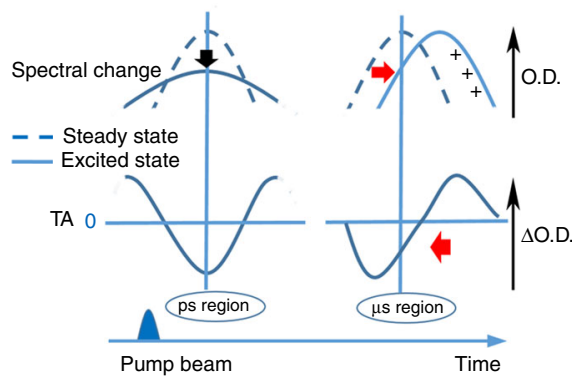

Fig. 2 Extinction spectra, band diagrams, TR-IR spectra, and a schematic illustration of time-dependent changes in TA. a Extinction spectra of CuS nanocrystals (NCs) and CdS/CuS heterostructured NCs (HNCs) in hexane. $\mathbf{b}$ Band diagrams of CuS and CdS phases, $E_{\mathrm{pF}}, E_{\mathrm{nF}}$ : Fermi level, the red arrow means plasmon excitation by near-infrared (NIR) light. c, d Time resolved IR spectroscopy (TR-IR) spectral changes for $\mathbf{c}$ CUS NCs and $\mathbf{d}$ CdS/CuS HNCs from visible to near-infrared regions in the microseconds ( $\mu$ s) region. e Kinetic profile of transient absorption (TA) for CdS/CuS HNCs at a specific probing wavelength $(560 \mathrm{~nm})$ tracking holes generated in the CdS phase by NIR local surface plasmon resonance (LSPR) excitation. Red line is the best fit. Pumping wavelength: $1200 \mathrm{~nm}$. The $\Delta$ mO.D. means the change of optical density. $\mathbf{f}, \mathbf{g}$ Schematic illustration of spectral changes at different timescales: $\mathbf{f}$ Bleaching of LSPR peak caused by hole and photon scattering in the CuS NCs observed in the ps region, and $\mathbf{g}$ surface hole trapping to reduce the number of holes in the intrinsic state, leading to a red-shift of the LSPR peak and blue shift of the bleaching position in the $\mu$ segion

cause a significant shift in the LSPR band ${ }^{17-19}$. Figures $2 \mathrm{f}$ and $\mathrm{g}$ show a schematic illustration of the spectral change and corresponding $\mathrm{TA}$ in different time regions. In the ps region, the aforementioned LSPR-excitation-induced sequential events caused LSPR bleaching (Fig. $2 \mathrm{f})^{11}$. When some of the hot holes were trapped at trapping sites, the number of holes in the intrinsic state decreased, causing the red-shift in the LSPR peak, which was responsible for the significant blue-shift of the bleaching position in the $\mu$ s region (Fig. 2g). The time-resolved blue-shift of bleaching signal was also clearly observed by using the nstransient absorption spectroscopy (See Supplementary Fig. 8). The red-shift of bleaching of the CdS/CuS HNCs in comparison with the CuS NCs is derived from the different LSPR position.

It is noteworthy that a broad and structureless absorption derived from the trapped holes in the CdS phases was observed as positive signal in the visible region ${ }^{20,21}$, providing direct evidence of LSPR-induced hole transfer from the CuS to CdS phase. The blue-shift of bleaching and the absorption of trapped hole of CdS makes significant difference between the CuS NCs and the CdS/ CuS HNCs in the transient absorption spectra at the timescale of $\mu$ s region (see detail explanation in Supplementary Fig. 8). As the decay of the trapped hole corresponds to charge recombination, the charge recombination rate between the CuS phase and CdS phase was estimated to be $1.1 \times 10^{5} \mathrm{~s}^{-1}$ (Fig. 2e), indicating that the charge separation was long-lived.

As the hot carrier generated in CuS NCs should decay within a few ps, TA measurement in the ps region is essential for elucidating the hot hole transfer mechanism. Therefore, we measured the TA of the CuS NCs and CdS/CuS HNCs in the ps region to investigate the LSPR-induced hot hole transfer mechanism (Fig. 3a). As the LSPR peak of the CuS phase is sensitive to carrier density ${ }^{18}$, the hole transfer can be determined using the time-resolved change in the LSPR band. As shown in Fig. 3a, the LSPR recovery upon excitation closely resembled the 

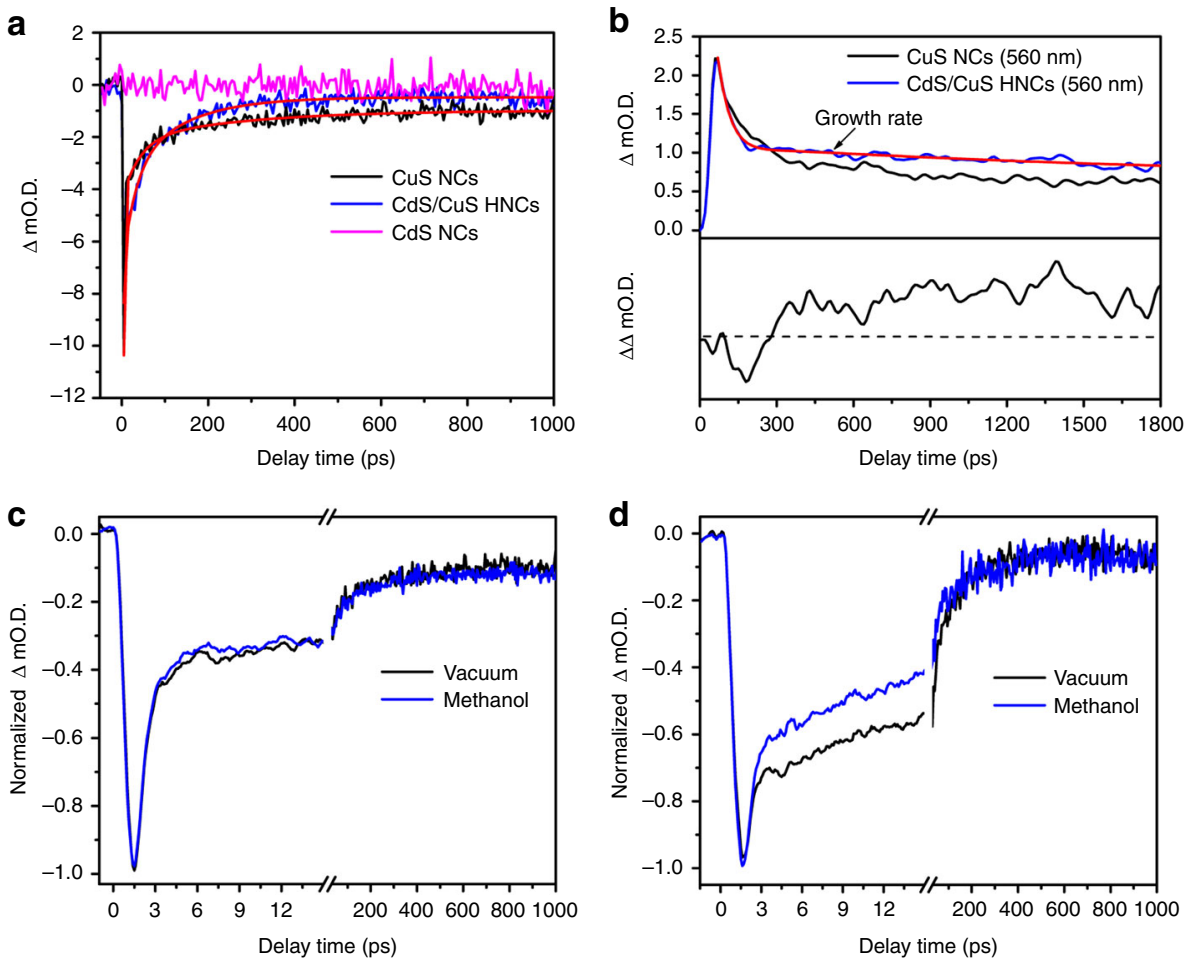

Fig. 3 Kinetic profiles and quenching study of nanocrystals after LSPR excitation. a Kinetic profiles of CuS nanocrystals (NCs), CdS NCs, and CdS/CuS heterostructured NCs (HNCs) at $1000 \mathrm{~nm}$; red line is the best fit. b Decay profiles of CuS NCs and CdS/CuS HNCs at $560 \mathrm{~nm}$; red line is the best fit. The rising component corresponds to the trapped holes in the CdS phase of CdS/CuS HNCs. c, d Quenching experiments by hole scavenger (methanol vapor: 20 Torr) for c CuS NCs and d CdS/CuS HNCs probing at $1000 \mathrm{~nm}$. The $\Delta$ mO.D. means the change of optical density. Excitation wavelength: $1200 \mathrm{~nm}$

laser pulse shape, followed by a slower recovery. A similar trend was previously observed in the TA measurements of CuS NCs by Cozzolli and coworkers, although slow components were not discussed $^{11}$. The LSPR bleaching and recovery of the CuS NCs at $1000 \mathrm{~nm}$ was well-fitted by a triexponential function with two clear components, $0.5 \mathrm{ps}\left(\tau_{1}\right)$ and $110 \mathrm{ps}\left(\tau_{2}\right)$, and one unclear component, for which the time constant $\left(\tau_{3}\right)$ could not be measured in the time frame of fs TR-IR (3 ns) (Supplementary Table 1). Since carrier trapping significantly affects LSPR recovery, we concluded that this multi-step recovery of LSPR reflected carrier-trapping-mediated relaxation, in addition to conventional LSPR decay. As the LSPR-mediated hole-trapping rate of $\mathrm{CuS} \mathrm{NCs}$ has been reported as $<2 \mathrm{ps}$, the fastest component corresponded to both ultrafast carrier trapping and a carrier-scattering-mediated decay process ${ }^{17}$. The decay components $\tau_{2}$ and $\tau_{3}$ corresponded to the relaxation process through hole trapping in deep and shallow states, as discussed below.

The kinetic trace of the LSPR bleaching and recovery of CdS/ CuS HNCs was also well-fitted by a triexponential function with components of $0.4 \mathrm{ps}\left(\tau_{1}\right), 80 \mathrm{ps}\left(\tau_{2}\right)$, and $>3 \mathrm{~ns}\left(\tau_{3}\right)$. The $\tau_{2}$ value of the CdS/CuS HNCs was smaller than that of the CuS NCs. We conducted quenching experiments using methanol as a hole scavenger to determine whether hole extraction affected the LSPR recovery. As shown in Fig. 3c, d, in the presence of methanol vapor, a slight increase in the recovery rate was observed for the CuS NCs. This feature was dramatically increased in the CdS/CuS HNCs. This change could be caused by the spectral change in the LSPR band due to the loss of holes. The dramatic increase in the LSPR band recovery rate for CdS/CuS HNCs by methanol reflected charge separation in the HNC, which led to efficient hole scavenging by methanol. Based on these results, it was conceivable that hole extraction from the $\mathrm{CuS}$ phase promoted LSPR recovery at $1000 \mathrm{~nm}$. Therefore, we concluded that the smaller $\tau_{2}$ value of the CdS/CuS HNCs, compared with that of the $\mathrm{CuS}$ NCs, reflected hole transfer from the CuS phase to the CdS phase. From the $\tau_{2}$ values of the CuS NCs and CdS/CuS HNCs, the hole transfer rate $\left(k_{2}\right)$ in the CdS/CuS HNCs was estimated to be $3.4 \times 10^{9} \mathrm{~s}^{-1}$.

The plasmon-induced hot hole transfer mechanism. Figure $3 \mathrm{~b}$ shows the kinetic profiles of CuS NCs and CdS/CuS HNCs at 560 $\mathrm{nm}$, tracing hole trapping in the CdS phase. The decay profile of the CuS NCs was well-fitted by a biexponential decay function. These components have been reported as trapped holes in the $\mathrm{CuS} \mathrm{NCs}{ }^{17}$. In contrast, the decay profile of CdS/CuS HNCs at $560 \mathrm{~nm}$ was not well-fitted by this function, but by a biexponential decay and a single exponential growth function, reflecting the evolution of absorption corresponding to the trapped holes in the CdS phases. As the hole trapping rate in the CdS NCs was faster than $1.4 \times 10^{12} \mathrm{~s}^{-1}$ (ref. ${ }^{21}$ ), the growth rate $\left(k_{\mathrm{r}}=5.6 \times\right.$ $10^{9} \mathrm{~s}^{-1}$ ) mainly reflected hole transfer from the CuS phase to the CdS phase (Supplementary Table 1). The agreement of the hole extraction rate from the CuS phase $\left(k_{2}\right)$ with the hole accepting rate of the trapped state $\left(k_{\mathrm{r}}\right)$ of the CdS phase strongly supported our hypothesis. The quantum efficiency of observed hole transfer was estimated to be $19 \%$ by using the following equation: $\Phi=\frac{n_{\mathrm{h}}}{N_{\text {photons }}}$, where $n_{\mathrm{h}}$ is the number of trapped holes generated in the CdS domain, and $N_{\text {photons }}$ is the number of photons absorbed by the CuS domain (see Supplementary Note 2 for detailed cal(ulation $)^{21}$.

It should be emphasized that the $\Phi$ of hole transfer is significantly high although hole transfer rate in the present system is much slower than the decay rate of hot holes (approximately $2 \times 10^{12} \mathrm{~s}^{-1}$ ). This contradiction strongly suggested that the hot holes were not directly injected into the CdS 
phase, but transferred stepwise via transit through the carrier trapping state. We have named this carrier transfer process plasmon-induced transit charge-transfer (PITCT). This mechanism was further confirmed by LSPR-induced hole transfer to a hole-scavenging organic molecule (triphenylamine (TPA)). In TPA-protected CuS NCs, hole transfer to TPA was observed at a rate of $3.3 \times 10^{10} \mathrm{~s}^{-1}$ after excitation of the LSPR band. Furthermore, the slower hole transfer rate to TPA compared with the decay rate of hot holes strongly supported the PITCT mechanism (Supplementary Figs. 9-13).

Furthermore, the theoretical calculation of mean free path of hot holes was conducted to support our hypothesis. By using the jellium model, the mean free path of hot holes in the CuS NCs was estimated to be $2.87 \mathrm{~nm}$ (see Supplementary Note 2 for detailed calculation) $)^{22,23}$. Taking the decay channel of holes trapping into consideration, the actual mean free path of hot holes should be shorter than the theoretically value estimated using the jellium model. Based on the conventional tunneling mechanism, only the hot holes generated within the region defined by the mean free path from the heterointerface of the $\mathrm{CdS} / \mathrm{CuS} \mathrm{HNCs}$ can participate effectively in interfacial hole transfer. Therefore, it is unlikely that the present hole transfer with an efficient $\Phi$ proceeds via the conventional mechanism. The short mean free path of hot holes strongly enforces the hole transfer via a PITCT mechanism.

To prove the contribution of PITCT to the IR responsive catalytic activity, we used methylene blue $(\mathrm{MB}$, oxidation potential of $0.523 \mathrm{~V}$ vs. $\mathrm{NHE}^{24}$ ) as a probe. The CdS/CuS HNCs exhibited oxidation catalytic activity superior to those of CuS NCs and CdS NCs under NIR light irradiation (Supplementary Fig. 14). The degradation kinetics were pseudo-first-order dynamics, so the reaction rate could be estimated using the following equation: $\ln \left(C / C_{0}\right)=-n \times t+b$, where, $C_{0}, C, n, t$ and $b$ are the initial $\mathrm{MB}$ concentration, the $\mathrm{MB}$ concentration in solution, the degradation rate constant of degradation, the reaction time, and the reaction constant, respectively. As shown in Supplementary Fig. 14, the $n$ value of CdS/CuS HNCs was 34 times higher than that of pristine CuS NCs due to the efficient hot hole extraction from $\mathrm{CuS}$ to the VB of CdS via PITCT, indicating that the PITCT process realized efficient NIR light-responsive catalytic activity.

\section{Discussion}

We have summarized the PITCT mechanism in Fig. 4. The hole transfer rate observed in this experiment $\left(3.4-5.6 \times 10^{9} \mathrm{~s}^{-1}\right)$ was much slower than the decay rate of hot holes in $\mathrm{CuS}$. This slow PITCT rate realized LSPR-induced hole transfer and a subsequent oxidation reaction using NIR light. We anticipate that PITCT could solve the problems of conventional LSPR-induced energy conversion, such as ultrafast relaxation of hot carriers and energy loss by charge recombination, and facilitate efficient energy conversion using low-energy IR light with plasmonic materials. Furthermore, the suitable band alignments and the defect sites play an important role for the long-lived charge separation with high quantum yields. PITCT adds a new dimension to optical materials science by controlling LSPR-induced carrier dynamics through defect engineering technology.

\footnotetext{
Methods

Synthesis of hexagonal plate-shaped $\mathbf{c v}$-CuS NCs. A mixture of copper (I) acetate $(0.123 \mathrm{~g}, 1 \mathrm{mmol})$, and oleylamine $(10 \mathrm{~mL})$ was degassed at $160^{\circ} \mathrm{C}$ for 30 min. A solution of sulfur $(0.048 \mathrm{~g}, 1.5 \mathrm{mmol})$ in 1-octadecene $(15 \mathrm{~mL})$ was injected rapidly into the mixture under a nitrogen atmosphere, and stirred for $10 \mathrm{~min}$. After the reaction, the resulting product was purified by adding ethanol-hexane $(v: v=$ $1: 1$ ) mixed solvent to the solution, centrifuging twice, and then redispersed the precipitate in hexane.
}

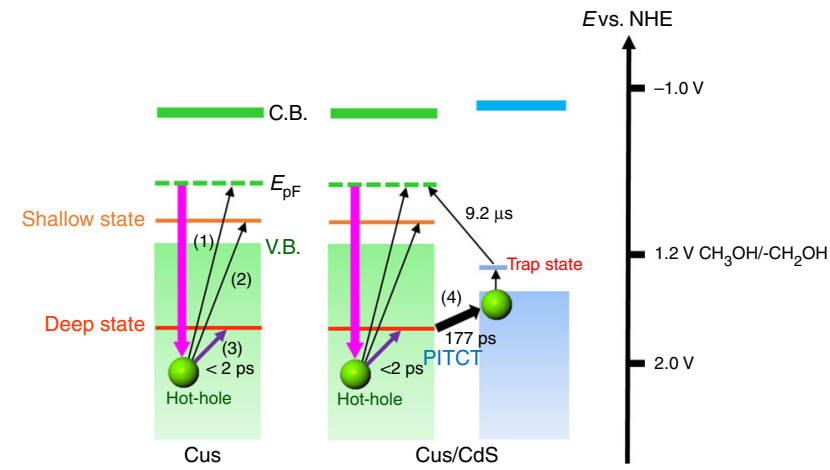

Fig. 4 Schematic illustration of LSPR-induced stepwise hole transfer process. Decay processes of hot holes generated in CuS NCs and CdS/CuS HNCs are shown. The red arrows means plasmon excitation by nearinfrared (NIR) light. For CuS NCs, the generated hot holes decayed via hole-hole and phonon-hole scattering (1) or ultrafast hole trapping to the shallow (2) or deep trapping state (3), followed by relaxation to the intrinsic hole state. In CdS/CuS heterostructured nanocrystals (HNCs), the holes in the deep trapping state transferred to the valence band (VB) of the CdS phases (4, PITCT) and the holes in the CdS phases moved to the trapping state, showing structureless absorption in the visible region and recombination to the initial state. PITCT: plasmon-induced transit carrier transfer

Synthesis of CdS/CuS HNCs. The metal thiocarbamate precursor of the hole acceptor phase (CdS) was prepared as follows: Sodium diethyldithiocarbamate (5 g $22 \mathrm{mmol})$ was dissolved in water $(100 \mathrm{~mL})$ and a solution of $\mathrm{Cd}\left(\mathrm{NO}_{3}\right)_{2} \cdot 4 \mathrm{H}_{2} \mathrm{O}$ $(3.085 \mathrm{~g}, 10 \mathrm{mmol})$ in water $(100 \mathrm{~mL})$ was added, followed by stirring for $1 \mathrm{~h}$. The resulting product was washed twice with water, centrifuged, washed again by ethanol, centrifuged and dried in an oven at $70^{\circ} \mathrm{C}$ for $12 \mathrm{~h}$ to obtain the Cd precursor. A mixed solution of CuS NCs $(0.2 \mathrm{mmol})$, oleylamine $(5 \mathrm{~mL})$, and 1octadecene $(10 \mathrm{~mL})$ was degassed at $150^{\circ} \mathrm{C}$ for $30 \mathrm{~min}$. A solution of the $\mathrm{Cd}$ precursor $(50 \mathrm{mg})$ in oleylamine $(2 \mathrm{~mL})$ was prepared with ultrasonication and injected into the above mixed solution under a nitrogen atmosphere at a rate of 0.1 $\mathrm{mL} \mathrm{min}^{-1}$, and stirred for a further $30 \mathrm{~min}$ after finishing the injection. The product was purified by centrifugation with hexane-ethanol $(v: v=1: 1)$ mixed solvent, and redispersed in hexane.

Characterization. Transmission electron microscopy (TEM) and high resolution TEM (HRTEM) characterizations were performed on JEM1011 (JEOL) and JEOL2200FS (equipped with Cs-corrector for TEM) electron microscopes with operating voltages of $100 \mathrm{kV}$ and $200 \mathrm{kV}$, respectively. HAADF-STEM and EDS-mapping were performed on a JEM-ARM200F (spherical aberration correction device) electron microscope with an operating voltage of $200 \mathrm{kV}$. The XRD patterns were recorded on a PANalytical X'Pert Pro MPD diffractometer, with $\mathrm{Cu}$ Ka radiation $(\lambda=1.542 \AA)$ at $45 \mathrm{kV}$ and $40 \mathrm{~mA}$. Ultraviolet-visible-near-infrared (UV-vis-NIR) absorption spectra were recorded using a U-4100 spectrophotometer (Hitachi). X-ray fluorescence (XRF) spectroscopy elemental analysis was carried out using Element Analyzer JSX-3202C (JEOL). ${ }^{1} \mathrm{H}$ NMR spectra were measured on JEOL JNMECP300 $(300 \mathrm{MHz})$ spectrometers. Matrix-assisted laser desorption ionization-time-of-flight mass spectrometry (MALDI-TOF MS) was performed on a Bruker Autoflex Speed instrument using trans-2-[3-(4-tert-butylphenyl)-2-methyl-2-propenylidene] malononitrile (DCTB) as the matrix.

Photocatalytic degradation of methylene blue. The photocatalytic activities of CuS NCs and CdS/CuS HNCs were evaluated using an NIR light source (NIR light power density, $40 \mathrm{~mW} \mathrm{~cm}^{-2}$ ). To fabricate the NIR-light, a $300-\mathrm{W}$ Xe light source (Cermax, Excelitas Technology) was irradiated through broadband dielectric mirrors (region, 750-1100 nm, R300-32J, THORLABS) to cut out light of $750-1100 \mathrm{~nm}$. Water-soluble NCs $(0.05 \mathrm{mmol})$ were suspended in water $(3 \mathrm{~mL})$ containing methylene blue (MB, $90 \mathrm{mg})$, and the suspension was sealed in a quartz cell. After different reaction times, a $0.6-\mathrm{mL}$ aliquot of the suspension was sampled into a plastic tube and centrifuged to remove the NCs. The remaining MB concentration was determined by the characteristic MB wavelength at $464 \mathrm{~nm}$ using UV-vis-NIR spectrophotometry.

Transient absorption measurements. The behavior of NIR-LSPR-generated holes was investigated using homemade femtosecond-to-second time-resolved spectrometers ${ }^{25}$. In the femtosecond-to-nanosecond region, experiments were 
performed using a conventional pump-probe technique based on a Ti-sapphire laser system (Spectra Physics, Solstice and TOPAS Prime; duration, $90 \mathrm{fs}$; wavelength, $800 \mathrm{~nm}$; repetition rate, $1 \mathrm{kHz}$ ). In this experiment, a $1200-\mathrm{nm}$ laser pulse was used as the pump pulse. For the microsecond measurements, in the visible-toNIR region, a halogen lamp $(50 \mathrm{~W})$ and an InGaAs detector were used as the light source and detector, respectively. Transient absorption spectra were measured from $500 \mathrm{~nm}$ to $1 \mu \mathrm{m}$. The spectra were obtained at 200-nm intervals and averaged over 300 scans for a spectrum by irradiating the sample using a pumping wavelength of $1200 \mathrm{~nm}$ generated by UV laser pulse from a Nd:YAG laser (Continuum, SureliteII; duration, $6 \mathrm{~ns}$; wavelength, $355 \mathrm{~nm}$; repetition rate, $0.01-5 \mathrm{~Hz}$ ). The powdery sample was fixed on a $\mathrm{CaF}_{2}$ plate with a density of $\sim 1 \mathrm{mg} \mathrm{cm}^{-2}$, and the sample plate was placed in a stainless-steel cell. Measurements were performed under vacuum at room temperature. For the ns-transient absorption measurements, the randomly-interleaved-pulse-train (RIPT) method was employed ${ }^{26}$. The pumppulse and probe source is a picosecond laser, PL2210A (EKSPLA, $1 \mathrm{kHz}, 25 \mathrm{ps}, 355$ $\mathrm{nm}, 0.3 \mathrm{~mJ}$ ), and a supercontinuum (SC) radiation source (SC-450, Fianium, 20 $\mathrm{MHz}, 50-100 \mathrm{ps}$ pulse width depending on the wavelength, $450-2000 \mathrm{~nm}$ ), respectively. A $1064 \mathrm{~nm}$-laser pulse was selected to excite the plasmon response. Generally, a chloroform solution of as-obtained samples in a $10 \mathrm{~mm}$-thick quartz cell under vigorously stirred was performed to do the ns-TA measurements at room temperature.

Data availability. The data sets within the article and Supplementary Information of the current study are available from the authors upon request.

Received: 18 August 2017 Accepted: 10 May 2018

Published online: 13 June 2018

\section{References}

1. Kalisman, P., Nakibli, Y. \& Amirav, L. Perfect photon-to-hydrogen conversion efficiency. Nano Lett. 16, 1776-1781 (2016).

2. Linic, S., Christopher, P. \& Ingram, D. B. Plasmonic-metal nanostructures for efficient conversion of solar to chemical energy. Nat. Mater. 10, 911-921 (2011).

3. Frischkorn, C. \& Wolf, M. Femtochemistry at metal surfaces: nonadiabatic reaction dynamics. Chem. Rev. 106, 4207-4233 (2006).

4. $\mathrm{Wu}, \mathrm{H}$.-L. et al. Formation of pseudomorphic nanocages from $\mathrm{Cu}_{2} \mathrm{O}$ nanocrystals through anion exchange reactions. Science 351, 1306-1310 (2016).

5. Trinh, T. T. et al. Visible to near-infrared plasmon-enhanced catalytic activity of Pd hexagonal nanoplates for the Suzuki coupling reaction. Nanoscale 7, 12435-12444 (2015).

6. Manjavacas, A., Liu, J. G., Kulkarni, V. \& Nordlander, P. Plasmon-induced hot carriers in metallic nanoparticles. ACS Nano 8, 7630-7638 (2014).

7. Hartland, G. V. Optical studies of dynamics in noble metal nanostructures. Chem. Rev. 111, 3858-3887 (2011)

8. Wu, K., Chen, J., McBride, J. R. \& Lian, T. Efficient hot-electron transfer by a plasmon-induced interfacial charge-transfer transition. Science 349, 632-635 (2015).

9. Clavero, C. Plasmon-induced hot-electron generation at nanoparticle/metaloxide interfaces for photovoltaic and photocatalytic devices. Nat. Photon. 8, 95-103 (2014).

10. Luther, J. M., Jain, P. K., Ewers, T. \& Alivisatos, A. P. Localized surface plasmon resonances arising from free carriers in doped quantum dots. Nat. Mater. 10, 361-366 (2011)

11. Xie, Y. et al. Metallic-like stoichiometric copper sulfide nanocrystals: phaseand shape-selective synthesis, near-infrared surface plasmon resonance properties, and their modeling. ACS Nano 7, 7352-7369 (2013).

12. Christopher, P., Xin, H. \& Linc, S. Visible-light-enhanced catalytic oxidation reactions on plasmonic silver nanostructures. Nat. Chem. 3, 467-472 (2011).

13. Saruyama, M. et al. Spontaneous formation of wurzite-CdS/zinc blende-CdTe heterodimers through a partial anion exchange reaction. J. Am. Chem. Soc. 133, 17598-17601 (2011).

14. Liu, Y., Liu, M. \& Swihart, M. T. Plasmonic copper sulfide-based materials: a brief Introduction to their synthesis, doping, alloying, and applications. J. Phys. Chem. C. 121, 13435-13447 (2017).

15. Brongersma, M. L., Halas, N. J. \& Nordlander, P. Plasmon-induced hot carrier science and technology. Nat. Nanotechnol. 10, 25-34 (2015).
16. Diroll, B. T., Guo, P., Chang, R. P. H. \& Schaller, R. D. Large transient optical modulation of epsilon-near-zero colloidal nanocrystals. ACS Nano 10, 10099-10105 (2016).

17. Ludwig, J. et al. Ultrafast hole trapping and relaxation dynamics in p-type $\mathrm{CuS}$ nanodisks. J. Phys. Chem. Lett. 6, 2671-2675 (2015).

18. Jain, P. K. et al. Doped nanocrystals as plasmonic probes of redox chemistry. Angew. Chem. Int. Ed. 52, 13671-13675 (2013).

19. Alam, R., Labine, M., Karwacki, C. J. \& Kamat, P. V. Modulation of $\mathrm{Cu}_{2-\mathrm{x}} \mathrm{S}$ nanocrystal plasmon resonance through reversible photoinduced electron transfer. ACS Nano 10, 2880-2886 (2016)

20. Wu, K., Du, Y., Tang, H., Chen, Z. \& Lian, T. Efficient extraction of trapped holes from colloidal CdS nanorods. J. Am. Chem. Soc. 137, 10224 (2015).

21. Wu, K. \& Lian, T. Quantum confined colloidal nanorod heterostructures for solar-to-fuel conversion. Chem. Soc. Rev. 45, 3781-3810 (2016).

22. Quinn, J. J. \& Ferrell, R. A. Electron self-energy approach to correlation in a degenerate electron gas. Phys. Rev. 112, 812 (1958).

23. Echenique, P. M., Pitarke, J. M., Chulkov, E. V. \& Rubio, A. Theory of inelastic lifetimes of low-energy electrons in metals. Chem. Phys. 251, 1-35 (2000).

24. Atamna, H. et al. Methylene blue delays cellular senescence and enhances key mitochondrial biochemical pathways. FASEB. J. 22, 703-712 (2008).

25. Yamakata, A. et al. Behavior and energy states of photogenerated charge carriers on Pt- or $\mathrm{CoO}_{\mathrm{x}}$-loaded $\mathrm{LaTiO}_{2} \mathrm{~N}$ photocatalysts: time-resolved visible to mid-infrared absorption study. J. Phys. Chem. C. 118, 23897-23906 (2014)

26. Nakagawa, T., Okamoto, K., Hanada, H. \& Katoh, R. Probing with randomly interleaved pulse train bridges the gap between ultrafast pump-probe and nanosecond flash photolysis. Opt. Lett. 41, 1498-1501 (2016).

\section{Acknowledgements}

This work was supported by JSPS KAKENHI Grant Numbers JP16H06520 (Coordination Asymmetry) (T.T.), JP17H05257 (Photosynergetics) (M.S.) and JP17H05491 (Mixed Anion) (A.Y.), and a JSPS Research Fellowship (17J09073) (Z.L.). The authors wish to thank Dr. D. Eguchi for synthesizing the TPA derivative molecules. We also thank T. Nakagawa for the ns-transient absorption measurement.

\section{Author contributions}

Z.L. and M.S. conceived, designed the whole experiments and carried out experiments and data analysis. T.T. supervised this study and provided intellectual and technical guideline. H.M., J.J.M.V., A.Y. carried out the TA experiments. M.H., H.K. carried out the high-resolution TEM and Elemental Mapping. W.O. and T.S. calculated the mean free path of hot hole. All authors participated in discussion of the research. Z.L., M.S., T. $\mathrm{T}$. wrote the paper.

\section{Additional information}

Supplementary Information accompanies this paper at https://doi.org/10.1038/s41467 018-04630-w.

Competing interests: The authors declare no competing interests.

Reprints and permission information is available online at http://npg.nature.com/ reprintsandpermissions/

Publisher's note: Springer Nature remains neutral with regard to jurisdictional claims in published maps and institutional affiliations.

pen Access This article is licensed under a Creative Common Attribution 4.0 International License, which permits use, sharing, adaptation, distribution and reproduction in any medium or format, as long as you give appropriate credit to the original author(s) and the source, provide a link to the Creative Commons license, and indicate if changes were made. The images or other third party material in this article are included in the article's Creative Commons license, unless indicated otherwise in a credit line to the material. If material is not included in the article's Creative Commons license and your intended use is not permitted by statutory regulation or exceeds the permitted use, you will need to obtain permission directly from the copyright holder. To view a copy of this license, visit http://creativecommons.org/ licenses/by/4.0/

(c) The Author(s) 2018 